\title{
A Cluster of FPAAs to Recognize Images Using Neural Networks
}

\author{
Daniel García Moreno (D), Alberto A. Del Barrio (D), Senior Member, IEEE, Guillermo Botella (D), Senior \\ Member, IEEE, and Jennifer Hasler (D), Senior Member, IEEE,
}

\begin{abstract}
Analog computing has been recovering its relevance in the recent years. FPAAs are the equivalent to FPGAs but in the analog domain. The main drawback of FPAAs is their reduced integration capacity. In order to increase the amount of analog resources, in this paper a cluster of 40 FPAAs is proposed. As a use case, a 19-8-6-4 feedforward Neural Network has been implemented on such cluster. With the help of a DCT-based software framework, this NN is able to classify $28 \times 28$ MNIST images. Results show that the analog network is able to obtain almost the same results as the software baseline network.
\end{abstract}

Index Terms-FPAA, cluster, neural network, analog computing, approximate computing, classification.

\section{INTRODUCTION}

C MOS scaling [1] is getting close to the physical limits, so it is mandatory to search for computing alternatives. According to Hennessy and Patterson [2], who received ACM Turing Award in 2018, the future is the heterogeneity within the systems and the use of approximate computing to increase the energy efficiency. The existence of applications that tolerate some margin of error, such as the Neural Networks (NNs), makes analog computing a good candidate to implement them [3][4]. Digital microprocessors dominate embedded application systems and general purpose computing [5], but it has been observed that the use of analog techniques may produce gains in terms of energy and area, compared with digital computation [6]. Analog computing can offer energy efficiency as well as the inherent behaviour of certain analog components. For example, their non-linearity can help solve differential equations efficiently [4].

As in the case of digital computation, there exist reprogrammable devices to prototype analog circuits. These devices are the Field Programming Analog Arrays (FPAAs). An FPAA [7] is a programmable chip that allows to deploy analog designs and reconfigure certain parameters and topologies. These programmable and configurable devices have the potential to transform low-power embedded systems [6][8]. The main drawback when employing currently available commercial

Manuscript received April 19, 2005; revised August 26, 2015. This work was supported by the CM under grant S2018/TCS-4423, the EU (FEDER) and the Spanish MINECO under grant RTI2018-093684-B-I00 and by Banco Santander under grant PR26/16-20B-1.

Daniel García Moreno, Alberto A. Del Barrio and Guillermo Botella are with the Department of Computer Architecture and Automation, Complutense University of Madrid, Madrid, 28040 Spain. (e-mail: \{daniel10,abarriog,gbotella\}@ucm.es).

Jennifer Hasler is with the School of Electrical and Computer Engineering (ECE), Georgia Institute of Technology, Atlanta, GA 30332-0250 USA. (email: jennifer.hasler@ece.gatech.edu).

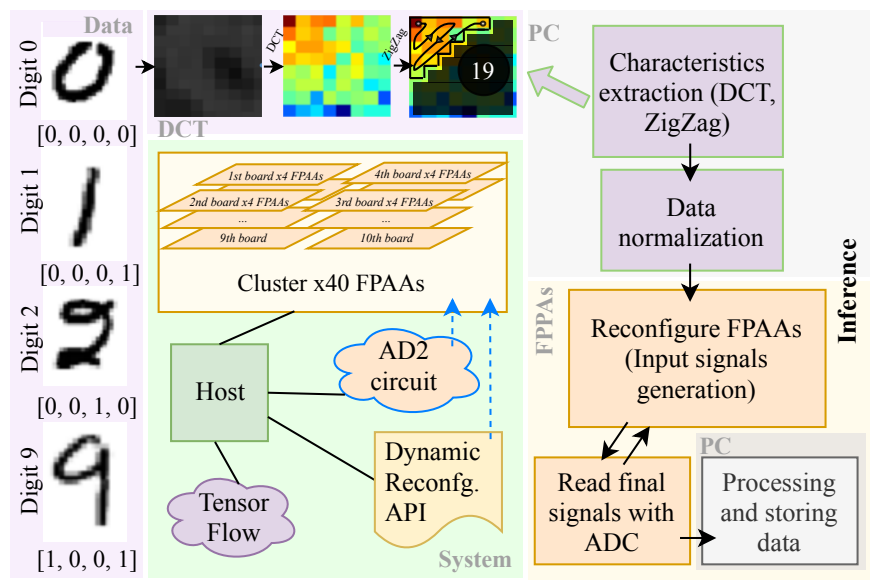

Fig. 1: Architecture of the FPAAs cluster and overview of the system.

FPAAs is their reduced integration capacity [9], [10], which makes it difficult to deploy large analog circuits on top of them. Furthermore, they do not possess many input-output elements (IOCells), so it is hard to process several data at the same time. For this reason, in this paper we propose the construction of a cluster of 40 FPAAs to increase the analog computation capability. As a Proof of Concept (PoC), a feedforward $\mathrm{NN}$ has been implemented employing the cluster. A $\mathrm{NN}$ is composed of neurons that essentially implement weighted sum operations followed by certain activation function. Deploying a NN in the analog domain implies utilizing analog neurons. Together with a software based featureextraction phase [11], this $\mathrm{NN}$ is able to process $28 \times 28$ images (MNIST database) [12], getting a similar accuracy as the equivalent fully software based NN. In this paper we propose to drive these features to the FPAAs cluster, by reconfiguring the gain of certain elements within the FPAAs employing their dynamic reconfiguration capabilities. The whole flow is shown in 1. In the following sections, the different elements will be detailed.

The rest of the paper is organized as follows: Section II details how the cluster has been built as well as the software support to perform inferences on 28x28 images; Section IIII shows the experimental results; Section IV] describes the literature about FPAAs and NNs and finally Section $\mathrm{V}$ gives our final remarks about this work. 


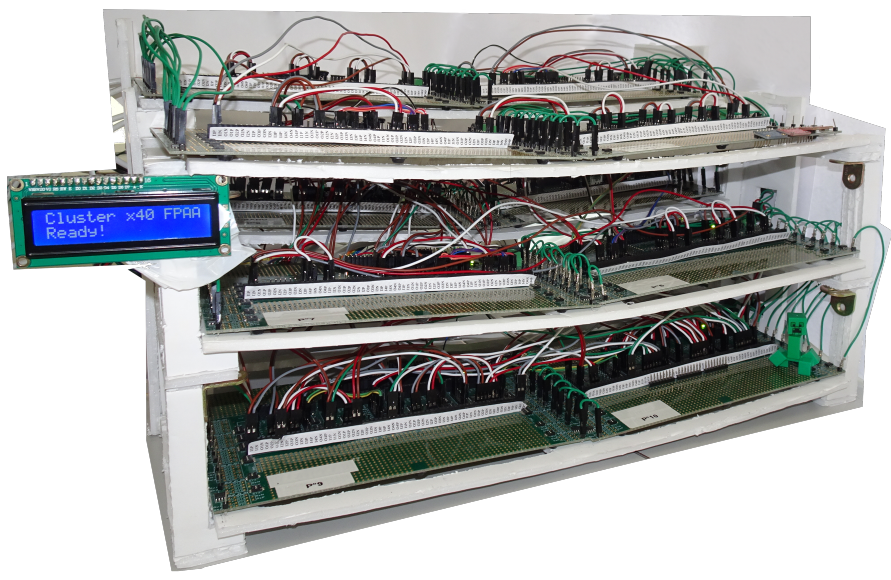

(a) Cluster of 40 FPAAs. The boards have been placed in a stacked fashion in order to minimize the size of the wires.

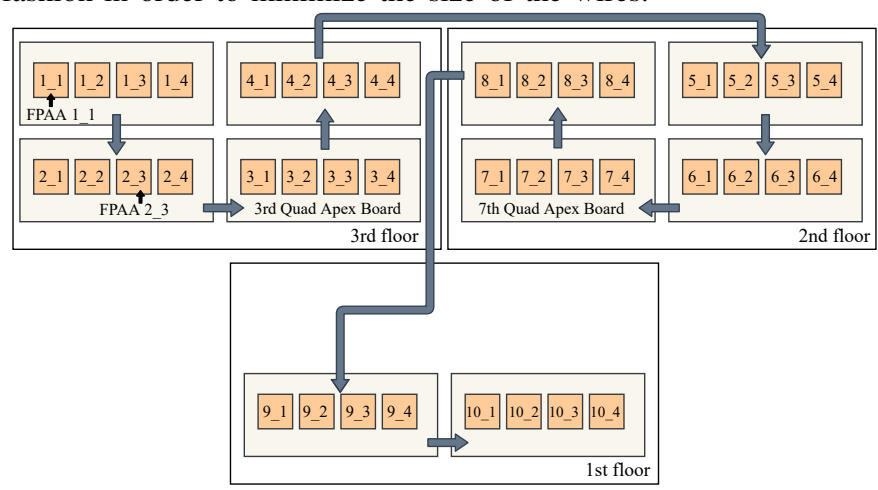

(b) Connection among the QuadApex boards. Each FPAA is identified by the label FPAAboardid_fpaaid. For instance, FPAA2_3 refers to the third FPAA belonging to the second QuadApex board.

Fig. 2: The experimental FPAA demonstration system.

\section{Cluster of FPAAs For Neural Networks}

In this section we present our cluster of FPAAs. The cluster has been constructed with 10 QuadApex V2.0 boards by Anadigm. Each of these boards has four AN231E04 FPAAs connected in a daisy-chain fashion and are managed by a microcontroller, which receives and sends data through the serial communication port. The cluster appearance can be seen in Fig. 2a. The FPAAs are wired to the adjacent ones with the aim of driving the signals according to the design. Fig. $2 \mathrm{~b}$ shows the diagram with the connection among the QuadApex boards. As it can be observed, the cluster is arranged in 3 floors. Each floor houses 4 Quad Apex V2.0 (except the first floor).

The analog circuits implemented on the cluster have been designed employing the Anadigm Designer 2 framework (AD2). This framework allows implementing analog designs using a library of Configurable Analogue Modules (CAMs) and can perform dynamic reconfiguration of parameters and topologies. This cluster is complemented by a host, as can be observed in the green box of Fig. 1. The host acts as controller of the whole system, sending the analog circuits to the cluster and interacting with the API generated through the AD2 framework to dynamically reconfigure the FPAAs.
As a $\mathrm{PoC}$ on the analog cluster, we have deployed a 198-6-4 feedforward NN whose neurons follow the MultiLayer Perceptron (MLP) style [9]. The neurons are responsible for making weighted sum operations of the outputs calculated in previous layers, followed by the application of an activation function. It must be noted that if $28 \times 28$ pixels were taken as entries, the NN would need to process 784 inputs overall. This approach typical of Convolutional Neural Networks (CNNs) as LeNet, would need hundreds of FPAAs. Thus, in order to minimize the resource consumption, only certain features have first been extracted through the DCT [11]. The NN identifies 10 different classes of $28 \times 28$ MNIST images. Every digit is identified by the binary representation of the digit. In order to find the NN architecture that best suits the cluster, making the most of the resources, leveraging the DCT transform and obtaining the highest possible accuracy, several configurations have been tested via software simulation. The number of outputs is given by the binary codification of the number of digits of MNIST (10 outputs) and the number of hidden layers has been fixed to two because of the resource constraints. The rest of parameters, i.e. inputs and neurons per layer, have been extensively simulated. As a result of this, the 19-8-6-4 network was selected as the best candidate.

It is important to note that every FPAA has 7 IOCells, which can be configured as input or output. This creates an important constraint when interconnecting not only the inputs and the outputs, but the neurons within the NN. As it has already been mentioned, the NN receives 19 DCT coefficients as inputs. Moreover, it possesses one hidden layer with 8 neurons, whose activation function is a sigmoid, a second hidden layer with 6 neurons and an output layer with 4 neurons, without the activation function part implemented. The MLP model needs a Transfer Function (TF) module to implement the neuron activation function. This is a component that uses a Lookup Table (LUT) to define its behavior. The utilization of the TF creates another constraint on the design, as there is only one LUT per FPAA. Hence, the theoretical maximum number of MLP neurons is the number of FPAAs, although this limit will not be reached because the resources are required for implementing other parts of the NN.

Although this cluster implementation is not optimal, it demonstrates two important characteristics. First, the cluster allows prototyping analog applications prior to being implemented through ASIC technologies. Second, this PoC proves one can build significant size analog NNs for a relevant image recognition, providing a benchmark for future developments.

Besides the hardware components, the system needs two software flows to train the $\mathrm{NN}$ and perform inferences, as it is illustrated in the rightmost part of Fig. 1. These will be described in detail in the following subsections.

\section{A. Training}

The training flow is responsible for configuring and training the NN, which has been done employing TensorFlow.

In order to diminish the number of inputs of the $\mathrm{NN}$, the $28 \times 28$ images go through the DCT. After applying the DCT, the 19 most significant coefficients are selected by employing 
the Zig Zag extraction method [11]. Once these coefficients are extracted, the 19-8-6-4 NN is trained using them as inputs of the network.

\section{B. Inference}

The inference flow is responsible for converting the characteristics of an image into analog signals, running the analog $\mathrm{NN}$ in the cluster of FPAAs and reading the final result of the NN using an Analog to Digital Converter (ADC).

1) Neuron implementation: To implement the MLP neuron model two elements are needed. On the one hand, a weighted adder must sum all neuron inputs, establishing weights for each input. This is done by configuring different gains for the adders. On the other hand, a TF is required in order to implement the activation function of the neuron.

It must be noted that the adder CAM has 4 inputs at most, however, all layers have a higher number of inputs. Hence, it is necessary to distribute the implementation of the neurons among multiple FPAAs. In order to utilize as few FPAAs as possible, each chip manages the weighted sum operations belonging to two neurons at the most. As can be seen in Fig. 3 . the components of two neurons " $X$ " and "Y" are highlighted with orange and dark green boxes respectively. In this figure, each input of the adders in FPAA3 4 corresponds to an input of the NN (wires labelled as n7, n8 and n9), except for one, that corresponds to the result of another weighted sum operation calculated in a prior FPAA (FPAA3_3) (wires labelled as n5 in the case of neuron " $\mathrm{X}$ " and $\mathrm{n} 6$ in the case of neuron "Y"). Finally, after computing the whole weighted sum, the $\mathrm{TF}$ module will perform the activation function. This CAM is shown in FPAA4_1, also highlighted with an orange box because it is the activation function of neuron "X". Due to resource limitations, the TF module of neuron "Y" must be allocated in another FPAA (FPAA4_2). The n12 wire carries the output value of the neuron "X", which will be the input of the following layer.

At the beginning of this section it was commented that the weight values are given by the adjustment of the adders gains. It is important to take into account that an adder CAM works in the range of $[-3 \mathrm{~V}, 3 \mathrm{~V}]$. If the $\mathrm{NN}$ has large weights, then large gains are set. Hence, any sum operation calculated on an adder CAM that generates a voltage that falls out of the previous range will cause a saturation in the weighted sum operation, interfering with the final classification decision of the NN. This problem has been solved in two steps. First, the established gains are not equal to the weights calculated in the training stage of the NN, but are divided by a scaling factor. Second, when the whole weighted sum operation is calculated, the final analog signal generated by the last adder is multiplied by the same scaling factor. Once this is done, the TF is applied and the output of the neuron is ready to be used by the following layer. One example of this implementation is shown in Fig. 3 In FPAA4_l the module in charge of multiplying the scaling factor is highlighted with an orange box and it is placed between $\mathrm{n} 10$ and $\mathrm{n} 11$ wires (placed between the last adder and the TF module).

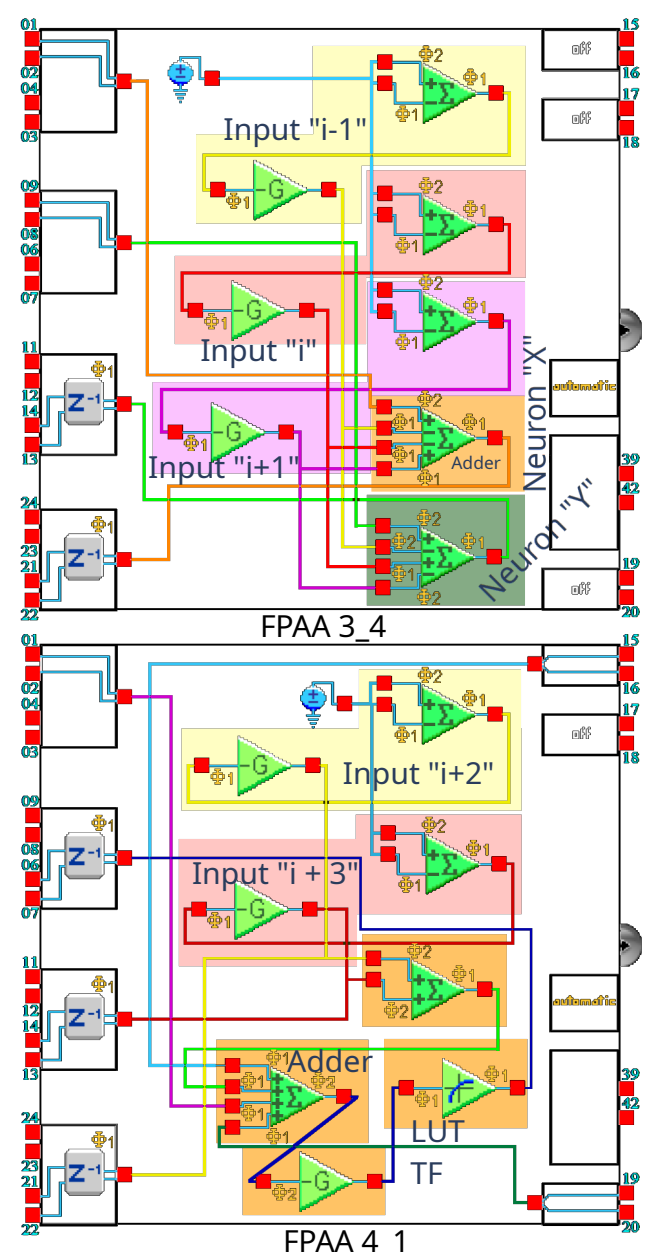

Fig. 3: Distributed implementation of the neurons.

2) Input management: In order to correctly perform the inference stage, it is necessary to provide 19 inputs in analog format to the NN. Our implementation proposes the internal generation of analog signals with a group of CAMs. This group is composed of one element that generates a constant $2 \mathrm{~V}$ signal, which is highlighted with a blue box in Fig. 3, as well as several adder-gain pairs, which are highlighted with yellow, red and purple boxes. It must be noted that the $2 \mathrm{~V}$ signal is driven to both inputs of the adders. The only difference among these pairs is the gain bound to the adders. This gain of the adder is then dynamically modified thanks to the dynamic reconfiguration capabilities of the FPAAs. The purpose of the gain block (labelled as $\mathrm{G}$ in the figure) is working as a phase converter to meet the phase requirements of the subsequent adders in the design.

This input management method allows us to get stable signals in the whole execution time of NN. In previous tests we observed that the use of a single DAC and several Sample and Hold modules degraded the signals very quickly, resulting in random internal and classification values.

\section{RESULTS}

In this section, the accuracy results of the NN during inference are shown. Two different NNs are compared. On the 


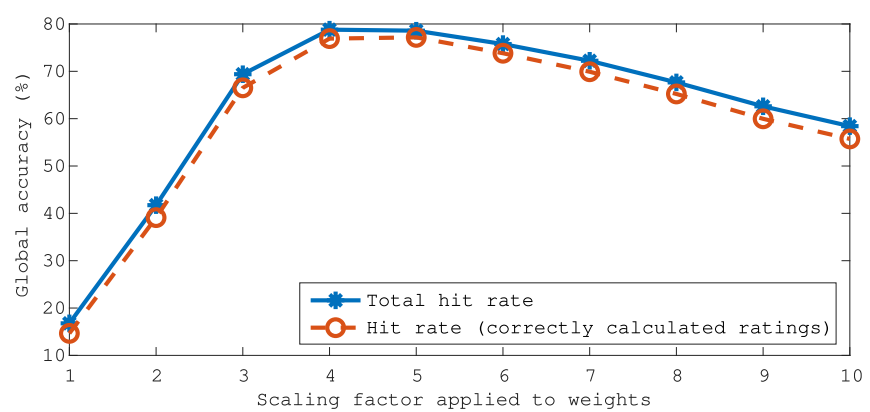

Fig. 4: Evolution of the hit rate as a function of the scaling factor applied. The blue line shows the total analog hit rate (including false positives). On the other hand, the red line shows the analog hits that match with the digital NN ones (correctly calculated classifications).

one hand, the baseline design is a software version of the 198-6-4 NN described in this work, which has been implemented with TensorFlow. On the other hand, the implemented analog $\mathrm{NN}$. The NN has been trained with the features extracted from 60000 images from the MNIST dataset and tested with other 10000 images.

\section{A. Feature extraction}

In order to extract the most significant features from the images, a transform is applied, followed by an algorithm to reduce the number of inputs of NN. From 784 inputs, 19 features are selected. In order to test the goodness of DCT when selecting features, other transforms have been tested, namely: DWT [13][14] and FFT [15]. These results are shown in Table II. As can be observed, the DCT extraction method obtains the best results, with an accuracy slighlty higher than $81 \%$. The performance of this NN in the analog domain using this transform will be detailed in the following paragraphs.

TABLE I: Tested transform algorithms.

\begin{tabular}{cccc}
\hline & \multicolumn{3}{c}{ Transforms } \\
\cline { 2 - 4 } & DWT & FFT & DCT \\
\hline Accuracy & $62.34 \%$ & $67.25 \%$ & $81.39 \%$ \\
\hline
\end{tabular}

\section{B. Scale factor selection}

In Section II-B1, the impact that the values of the weights have in the final result of the analog NN was mentioned. Therefore, in order to find the best scaling factor for this task, several values have been tested. Fig. 4, shows the evolution of the analog NN accuracy as a function of the applied scaling factor. It can be noted that the best scaling factor value is between 4 and 5 . However, the highest number of correctly classifications in the analog domain is achieved with a scaling factor value equal to 5. Therefore, in the rest of the experiments we will utilize this scaling factor.
TABLE II: Accuracy results of boths NNs

\begin{tabular}{ccccc}
\hline & DIGITAL & \multicolumn{2}{c}{ ANALOG } \\
\cline { 2 - 4 } Accuracy & & Accuracy & Match \\
\hline Digit 0 & $89.08 \%$ & & $89.18 \%$ & $99.19 \%$ \\
Digit 1 & $95.77 \%$ & & $95.41 \%$ & $98.98 \%$ \\
Digit 2 & $84.39 \%$ & & $86.04 \%$ & $99.08 \%$ \\
Digit 3 & $76.13 \%$ & & $76.33 \%$ & $97.91 \%$ \\
Digit 4 & $85.33 \%$ & & $87.37 \%$ & $99.16 \%$ \\
Digit 5 & $70.51 \%$ & & $71.18 \%$ & $97.29 \%$ \\
Digit 6 & $87.47 \%$ & & $87.57 \%$ & $98.32 \%$ \\
Digit 7 & $82.87 \%$ & & $78.11 \%$ & $94.01 \%$ \\
Digit 8 & $64.37 \%$ & & $56.36 \%$ & $85.32 \%$ \\
Digit 9 & $74.82 \%$ & $55.30 \%$ & $73.64 \%$ \\
\hline \hline & & \multicolumn{2}{c}{ GLOBAL ACCURACY } \\
DIGITAL & \multicolumn{2}{c}{$81.39 \%$} \\
ANALOG & $78.58 \%(77.17 \%$ correctly calculated) \\
\hline
\end{tabular}

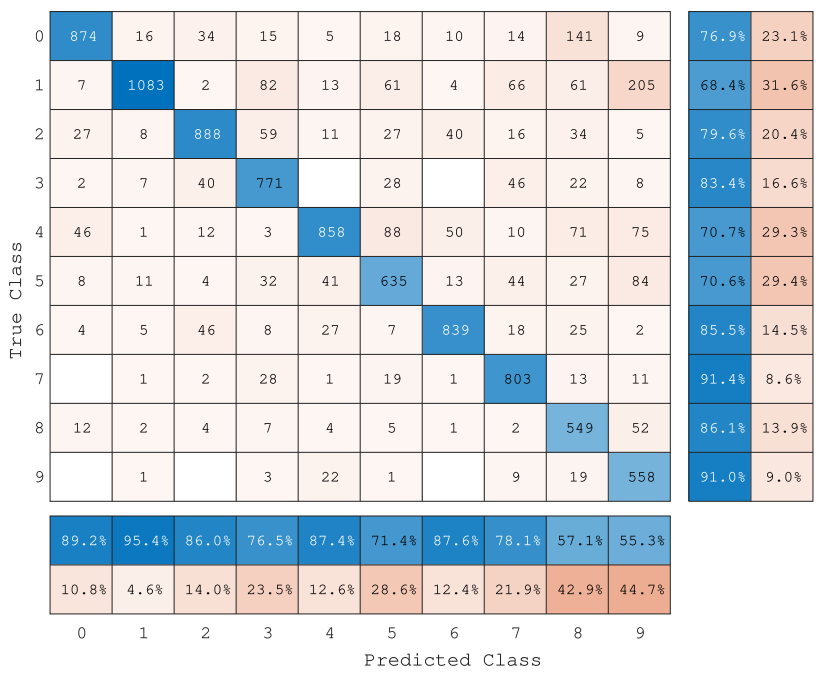

Fig. 5: Confusion Matrix.

\section{Digital and analog NN comparison}

Table II contains the data about the accuracy of the digital and analog NNs. The Accuracy columns show the hit percentage for both the digital and analog domains, while the Match column refers to the percentage of analog hits that coincide with their digital counterpart.

The overall digital accuracy is $81.39 \%$. It must be noted that this is a feedforward 19-8-6-4 network, much simpler in comparison with CNNs as LeNet. The analog NN achieved an overall accuracy close to $79 \%$. As can be observed, all digits but 8 and 9 reach $94-99 \%$ percentage matches. The case of digits 8 and 9 is troublesome for both NNs, but accuracy degrades more for the analog one.

Fig. 5 shows the confusion matrix. The accuracy is quite similar to that shown in Table $\Pi$. There are some differences because of the binary nature output of the $\mathrm{NN}$, producing several outputs that actually do not exist as a class, i.e. binary values greater than 1001 .

\section{RELATED WORK}

In the last years, the use of FPAAs has been increased in literature. For example, in [16] a Low Power Speech Detector was implemented for portable and remote sensing 
applications. In [17] a system for conditioning ECG signals was presented. Another scenario where FPAAs have been employed is the implementation of a Vector-Matrix Multiplier (VMM) and Winner-Take-All (WTA) classifier [18][19]. The recent generation of FPAAs show improved energy, area and bandwidth over commercially available FPAA devices, and the application mentioned in this paper would be an excellent demonstration on these newer platforms.

In addition to the aforementioned applications, FPAAs have also been utilized to implement NNs. In [9], authors implemented several types of neurons using analog components. The first model is the MCCulloch-Pitts. This model uses an adder to implement the weighted additions and a comparator to realize the activation function. Different gains are applied to each adder input, which work as weights within the neural network. The MLP model uses a Voltage Transfer function module to implement the activation function. Finally, the Spiking model implements its activation function thanks to an integrator with reset functionality. In this work, the MLP model has been employed. Section II explains how it has been implemented.

In several projects [9], [10], [20], the FPAAs were used to build NNs with 3 or 4 neurons and few inputs using the abovementioned models, but they all have in common that the few available resources prevents creating more complex NNs. In [21] a cluster of 5 AN221E04 FPAAs was used to build a NN employing a piece wise linear activation function. Authors constructed a NN with 2 inputs, 5 neurons in the hidden layer and 1 output (2-5-1) to classify two data types.

In order to solve the aforementioned limitations and deal with more complex designs, the cluster of 40 AN231E04 FPAAs has been constructed. This increase in the analog computation capability with respect to a single chip or QuadApex board has allowed us, as PoC, to develop a NN consisting of 19 inputs, 8 neurons on the first hidden layer, 6 neurons on the second hidden layer and 4 neurons on the output layer, increasing the size than the ones the literature shows.

\section{CONCLUSION}

In this paper a 40-FPAAs cluster has been constructed. As a PoC, an analog feedforward NN has been deployed achieving an accuracy very close to the software version. Furthermore, with the help of a DCT-based software framework, the proposed NN has been able to process $28 \times 28$ MNIST images, all in all, constituting an important advance with respect to the state-of-the-art FPAA-based NNs.

Future works can tackle the portability of the prototyped analog circuits into platforms that cram more CABs per inch [6] and/or ASICs. By applying a similar methodology, it will be possible to efficiently perform image processing tasks through NNs and analog hardware.

\section{ACKNOWLEDGMENT}

This paper has been supported by the $\mathrm{CM}$ under grant S2018/TCS-4423, the EU (FEDER) and the Spanish MINECO under grant RTI2018-093684-B-I00 and by Banco Santander under grant PR26/16-20B-1.

\section{REFERENCES}

[1] M. Anis, M. Allam, and M. Elmasry, "Impact of technology scaling on cmos logic styles," IEEE Transactions on Circuits and Systems II: Analog and Digital Signal Processing, vol. 49, no. 8, pp. 577-588, 2002.

[2] J. L. Hennessy and D. A. Patterson, "A new golden age for computer architecture," Commun. ACM, vol. 62, no. 2, p. 48-60, Jan. 2019. [Online]. Available: https://doi.org/10.1145/3282307

[3] R. S. Amant, A. Yazdanbakhsh, J. Park, B. Thwaites, H. Esmaeilzadeh, A. Hassibi, L. Ceze, and D. Burger, "General-purpose code acceleration with limited-precision analog computation," in 2014 ACM/IEEE 41st International Symposium on Computer Architecture (ISCA), June 2014, pp. 505-516.

[4] N. Guo, Y. Huang, T. Mai, S. Patil, C. Cao, M. Seok, S. Sethumadhavan, and Y. Tsividis, "Energy-efficient hybrid analog/digital approximate computation in continuous time," IEEE Journal of Solid-State Circuits, vol. 51, no. 7, pp. 1514-1524, July 2016.

[5] A. A. Del Barrio, R. Hermida, and S. Ogrenci-Memik, "A combined arithmetic-high-level synthesis solution to deploy partial carry-save radix-8 booth multipliers in datapaths," IEEE Transactions on Circuits and Systems I: Regular Papers, vol. 66, no. 2, pp. 742-755, 2019.

[6] J. Hasler, "Large-scale field-programmable analog arrays," Proceedings of the IEEE, vol. 108, no. 8, pp. 1283-1302, 2020.

[7] F. Baskaya, D. V. Anderson, and S. K. Lim, "Net-sensitivity-based optimization of large-scale field-programmable analog array (fpaa) placement and routing," IEEE Transactions on Circuits and Systems II: Express Briefs, vol. 56, no. 7, pp. 565-569, 2009.

[8] C. Mead, "Neuromorphic electronic systems," Proceedings of the IEEE, vol. 78, no. 10, Oct 1990.

[9] P. Rocke, J. Maher, and F. Morgan, "Platform for intrinsic evolution of analogue neural networks," vol. 2005, 10 2005, pp. 8 pp. - 11 .

[10] J. Maher, B. M. Ginley, P. Rocke, and F. Morgan, "Intrinsic hardware evolution of neural networks in reconfigurable analogue and digital devices," in 2006 14th Annual IEEE Symposium on Field-Programmable Custom Computing Machines, April 2006, pp. 321-322.

[11] D. Fariña, G. Botella, and A. A. D. Barrio, "A det and neural network based system to obtain the characteristics of biological images," in Proceedings of the Summer Simulation Multi-Conference, ser. SummerSim '17. San Diego, CA, USA: Society for Computer Simulation International, 2017.

[12] Y. Lecun, L. Bottou, Y. Bengio, and P. Haffner, "Gradient-based learning applied to document recognition," Proceedings of the IEEE, vol. 86, no. 11, pp. 2278-2324, 1998.

[13] B. Usman and A. Mustapha, "Satellite imagery dimensionality reduction using discrete wavelet transform for the extraction of cadastral features," vol. 12, 112015 .

[14] D. R. Nayak, R. Dash, and B. Majhi, "Brain mr image classification using two-dimensional discrete wavelet transform and adaboost with random forests," Neurocomput., vol. 177, no. C, p. 188-197, Feb. 2016. [Online]. Available: https://doi.org/10.1016/j.neucom.2015.11.034

[15] D. Soni, Y. K. Gupta, and S. Dubey, "Empirical study of dwt and fft techniques to extract intensity based features from the images," vol. 3, pp. 437-443, 102016.

[16] S. Shah and J. Hasler, "Low power speech detector on a fpaa," in 2017 IEEE International Symposium on Circuits and Systems (ISCAS), May 2017, pp. 1-4.

[17] D. P. Morales, A. García, A. J. Palma, and U. Meyer-Baese, "Fpaa suitability as analog front-end for biosignals," in Proc. SPIE 7674@, Smart Biomedical and Physiological Sensor Technologies VII@, 76740B, 04.

[18] S. Shah and J. Hasler, "SoC FPAA hardware implementation of a vmm+wta embedded learning classifier,' IEEE Journal on Emerging and Selected Topics in Circuits and Systems, vol. 8, no. 1, pp. 28-37, March 2018.

[19] J. Hasler and S. Shah, "An soc fpaa based programmable, ladder-filter based, linear-phase analog filter,' IEEE Transactions on Circuits and Systems I: Regular Papers, vol. 68, no. 2, pp. 592-602, 2021.

[20] B. Mc Ginley, P. Rocke, F. Morgan, and J. Maher, "Reconfigurable analogue hardware evolution of adaptive spiking neural network controllers," 01 2008, pp. 289-290.

[21] Puxuan Dong, G. L. Bilbro, and Mo-Yuen Chow, "Implementation of artificial neural network for real time applications using field programmable analog arrays," in The 2006 IEEE International Joint Conference on Neural Network Proceedings, July 2006, pp. 1518-1524. 